\title{
Timeline and Location of Recurrence Following Successful Ablation in Barrett's Esophagus: An International Multicenter Study
}

\begin{abstract}
Sarmed S. Sami ${ }^{1,7}$, Adharsh Ravindran ${ }^{1}$, Allon Kahn², Diana Snyder ${ }^{2}$, Jose Santiago ${ }^{3}$, Jacobo Ortiz-Fernandez-Sordo ${ }^{3}$, Wei Keith Tan ${ }^{4}$, Michael Heckman ${ }^{5}$, Ross Dierkhising ${ }^{6}$, Michele Johnson ${ }^{1}$, Ramona Lansing ${ }^{1}$, Kenneth Wang ${ }^{1}$, Krish Ragunath ${ }^{3}$, Massimiliano di Pietro ${ }^{4}$, Herbert Wolfsen ${ }^{5}$, Francisco Ramirez², David Fleischer ${ }^{2}$, Cadman L. Leggett ${ }^{1}$, David Katzka ${ }^{1}$, Prasad G. Iyer ${ }^{1}$
\end{abstract}

${ }^{1}$ Barrett's Esophagus Unit, Division of Gastroenterology \& Hepatology, Mayo Clinic, Rochester, MN, USA.

2Division of Gastroenterology \& Hepatology, Mayo Clinic, Scottsdale, Arizona, USA.

${ }^{3}$ National Institute for Health Research (NIHR) Biomedical Research Center in Gastrointestinal and Liver Diseases at Nottingham University Hospitals NHS Trust and the University of Nottingham, Queens Medical Center Campus, Nottingham, UK. ${ }^{4}$ Cambridge University Hospitals NHS Trust and MRC Cancer Unit, Hutchinson/MRC Research Center, University of Cambridge, Cambridge, UK.

${ }^{5}$ Division of Gastroenterology \& Hepatology, Mayo Clinic, Jacksonville, Florida, USA.

${ }^{6}$ Division of Biomedical Statistics and Informatics, Mayo Clinic, Rochester, USA.

${ }^{7}$ Division of Surgery and Interventional Science, University College London, London, UK. 


\section{Corresponding Author:}

Prasad G. Iyer, MD, MS

Professor of Medicine

Barrett's Esophagus Unit, Division of Gastroenterology and Hepatology

Mayo Clinic, 200 First Street SW, Rochester, MN 55905

Email: iyer.Prasad@mayo.edu

Phone: 507-255-6930

Fax: $507-255-7612$

Word Count: 3077

\section{Keywords:}

Barrett's esophagus, Ablation, Esophageal adenocarcinoma, Recurrence, Timeline, Predictors 
Sami, et al. 3

\section{Abstract}

Objectives: Surveillance intervals protocols after complete remission of intestinal metaplasia (CRIM) post radiofrequency ablation (RFA) in Barrett's esophagus (BE) are currently empiric and not based on substantial evidence. We aimed to assess the timeline, location, and patterns of recurrence following CRIM to inform these guidelines.

Design: Data on patients undergoing RFA for BE were obtained from prospectively maintained databases of five (three United States and 2 United Kingdom) tertiary referral centers. RFA was performed till CRIM was confirmed on two consecutive endoscopies.

Results: 594 patients achieved CRIM as of May $1^{\text {st }}$ 2017. 151 subjects developed recurrent BE over a median (IQR) follow up of 2.8 (1.4-4.4) years. There was $19 \%$ recurrence risk of any BE within 2 years and an additional $49 \%$ risk over the next 8.6 years. The recurrence hazard rate of any $\mathrm{BE}$, dysplastic $\mathrm{BE}$, and high grade dysplasia/cancer remained constant over the duration of follow-up ( $p=0.74, p=0.94$, and $p=0.88$; respectively). $74 \%$ of $B E$ recurrences developed at the gastroesophageal junction (GEJ) (24.1\% were dysplastic) and $26 \%$ in the tubular esophagus. The yield of esophageal random biopsies from the tubular esophagus, in the absence of visible lesions, was $1 \%(\mathrm{BE})$ and $0.2 \%$ (any dysplasia recurrence).

Conclusions: BE recurrence risk following CRIM remained constant over time, suggesting that lengthening of follow up intervals, at least in the first five years after CRIM, may not be advisable. Sampling the GEJ is critical to detecting recurrence. The requirement for random biopsies of the neo-squamous epithelium in the absence of visible lesions may need to be re-evaluated. 
What is already known about this subject?

- Recurrence rates of intestinal metaplasia and dysplasia following successful ablation for Barrett's esophagus are well established.

- Recent data suggests that rates of recurrence are highest in the initial year after remission and may decline thereafter suggesting widening of surveillance intervals after the initial year.

- Data on the timeline and endoscopic patterns of recurrence are scarce.

What are the new findings?

- Recurrence rates of intestinal metaplasia and dysplasia appear to increase progressively with time with no plateauing, in this multicenter international cohort study,

- Yield of neo-squamous epithelium biopsies from the tubular esophagus in the absence of visible recurrences is very low $(<1.0 \%)$.

- Most recurrences occur at the gastroesophageal junction (GOJ) or in the distal 5 $\mathrm{cm}$ of the esophagus

How might it impact on clinical practice in the foreseeable future?

- Widening of surveillance intervals to detect recurrence after BE ablation may be premature in the absence of additional data.

- Practice guidelines recommending Seattle protocol biopsies in the entire neosquamous epithelium in the absence of visible recurrences may need to be reevaluated. 
Sami, et al. 5

\section{Introduction}

Barrett's esophagus $(\mathrm{BE})$ is a condition that develops when the normal squamous epithelium is replaced by columnar mucosa with specialized intestinal metaplasia (IM). This process confers an increased risk of progression to esophageal adenocarcinoma (EAC) with an estimated incidence rate of approximately 0.33 per patient year. ${ }^{1}$ This risk increases significantly when either low grade dysplasia (LGD) or high grade dysplasia (HGD) develop in the setting of BE. ${ }^{2}$ Radiofrequency ablation (RFA) after endoscopic resection of visible lesions has been shown to significantly reduce the risk of cancer progression in patients with both LGD and HGD and has therefore become the standard of care in those patients. ${ }^{2}$ RFA is deemed to be successful once complete remission of intestinal metaplasia (CRIM) is achieved both endoscopically and histologically. However, recurrence of both IM and dysplasia occurs, with an estimated annual incidence rate of $9.5 \%$ for any recurrence and $2 \%$ for dysplastic recurrences. ${ }^{3,4}$ For these reasons, guidelines recommend regular surveillance every 3 months for the first year following CRIM, then every 6 months for the second year and then yearly afterwards. ${ }^{2}$

Data from a recent systematic review suggest that recurrence rate may be significantly higher in the first year compared to subsequent years, raising the question, whether surveillance intervals should be extended to every 2 or 3 years instead of yearly after CRIM. ${ }^{5}$ However, this difference was not noted in the subgroup of studies defining CRIM more stringently as two negative endoscopies $(n=3) \cdot{ }^{6-8}$ The finding of columnar mucosa or IM on histology after one negative endoscopy may represent incompletely treated or missed prevalent disease rather than true recurrence and may therefore overestimate the recurrence rate. ${ }^{5}$ None of the latter studies evaluated the variation in the incidence of 
recurrence over time across all grades of dysplasia. Moreover, other studies have thus far been limited by either small sample size (of CRIM patients with small number of dysplastic recurrences) ${ }^{6,9,10}$ or the inclusion of large number of patients treated with no dysplasia whose recurrence patterns may not be reflective of those with dysplasia. ${ }^{11}$ Hence, there is currently no conclusive evidence to demonstrate that the recurrence rate following CRIM remains constant over time (new cases developing every year) in order to justify currently recommended yearly surveillance or whether the rate plateaus after some time (no new cases developing) which may therefore justify widening surveillance intervals.

Guidelines recommend that during surveillance after CRIM, random biopsies should be obtained from the gastroesophageal junction (GEJ) and the neo-squamous epithelium at $1-2 \mathrm{~cm}$ intervals to cover the extent of the previous BE segment. ${ }^{2}$ Longitudinal data on the yield of this approach, particularly, in the absence of endoscopically visible recurrence are limited. Such data will be valuable in determining the cost effectiveness of this practice and in informing future guidelines. Similarly, the location of recurrence in the tubular esophagus needs to be better defined in order to justify surveillance biopsies over the entire length of the previous BE segment.

Given these knowledge gaps, we aimed to assess the timeline, location, and patterns of recurrence following CRIM in a large multicenter, and international cohort with the goal of informing future guidelines for endoscopic surveillance after CRIM. We also used a conservative definition of CRIM as two consecutive negative endoscopies with biopsies (from the esophagus and GEJ) for the reasons detailed above.

\section{Materials and Methods}




\section{Study design}

This was a cohort study of patients undergoing RFA for BE in five tertiary referral centers with expertise in the management of this condition. Three centers were located in the United States (Mayo Clinic Rochester, Mayo Clinic Arizona, and Mayo Clinic Florida) and 2 in the United Kingdom (Nottingham and Cambridge University Hospitals). The study was approved by the Institutional Review Boards of the respective centers. Data were obtained from prospectively maintained databases at each of the participating centers. We included patients who are 18 years or older with endoscopically (at least $1 \mathrm{~cm}$ of columnar mucosa in the tubular esophagus) and histologically (presence of IM) confirmed BE with or without dysplasia, who underwent RFA. Patients with advanced cancer stage (T2 or higher), pregnancy, or esophageal varices were excluded from the study. RFA procedures were performed between November 2003 and July 2016. All patients included in this study achieved CRIM between March 2004 and May 2017 and had at least one follow up endoscopy to check for recurrence. The study was conducted and reported according to the STROBE guidelines ${ }^{12}$.

\section{Participants and Interventions}

Patients underwent RFA by expert endoscopists following endoscopic assessment using high definition white light endoscopy and narrow band imaging with or without endoscopic resection of any visible lesions. Both circumferential and focal RFA was used to treat the BE segments as well as the GEJ. Energy settings followed manufacturer recommendations and RFA was performed every 3 months till CRIM was achieved. CRIM was defined as two consecutive endoscopies at least 3 months apart confirming the absence of IM on biopsies from both the GEJ (top of the gastric folds within $1 \mathrm{~cm}$ of the 
neo-squamo-columnar junction) and tubular esophagus. In addition to RFA, patients could receive argon plasma coagulation or multipolar coagulation, as rescue (adjuvant) techniques for minimal residual BE islands.

\section{Post CRIM surveillance protocol}

Once CRIM was achieved, subsequent surveillance was performed at 3, 6, 9, and 12 months thereafter. All patients underwent high definition white light endoscopy and narrow band imaging with random biopsy specimens obtained from the GEJ and every 1 2 centimeters in 4 quadrants to cover the area of the previous BE segment as a minimum requirement. Samples from each level were labelled and stored in separate bottles. In addition, targeted biopsies were taken from any visible lesions (both columnar and/or neosquamous). Recurrence was defined as the histologic presence of IM with or without dysplasia on biopsy specimens taken from either the tubular esophagus or the GEJ or both after CRIM was achieved. The location, visibility, and the dysplasia status of all recurrences were documented. A separate sensitivity analysis was performed for recurrence incidence rate after excluding non-dysplastic BE (NDBE) recurrences at the GEJ given concern that this may represent residual IM or IM of the cardia rather than true BE recurrence. ${ }^{13}$

\section{Histology}

Baseline histology was recorded for all patients upon entry into the study and classified as: non-dysplastic BE (NDBE); indefinite for dysplasia (ID); LGD; HGD; and cancer. Biopsy specimens at each center were examined by an expert gastrointestinal pathologist. The worst grade of dysplasia detected on tissue sampling at baseline (preRFA) and at post-CRIM surveillance (post-RFA) was assigned to that patient. 
Sami, et al. 9

\section{Statistical Analysis}

The Kaplan-Meier method was used to estimate the cumulative incidence of recurrence after CRIM. Tests of a constant recurrence hazard rate were performed with likelihood ratio tests comparing exponential vs. Weibull distributions for time to recurrence. Cox proportional hazards models were used to measure associations of a priori-set baseline variables with recurrence. Multivariable Cox models were used to estimate adjusted effects of each variable on recurrence, with Firth estimation in cases with low recurrence totals. A separate sensitivity analysis was performed for recurrence incidence rate after excluding NDBE recurrences at the GEJ given concern in some studies that this may represent residual IM or IM of the cardia rather than true BE recurrence. ${ }^{13}$

\section{Results}

\section{Baseline Characteristics}

594 patients achieved CRIM as of May $1^{\text {st }} 2017$ and were included in the analysis (Table 1). Figure 1 shows the patient flow chart. Mean (standard deviation (+/-SD)) age was 67 $(+/-10)$ years and $86 \%$ were males. Median (interquartile range (IQR)) BE segment length was $4(2-6) \mathrm{cm} .90 \%$ of patients were treated for dysplasia or carcinoma.

\section{Table 1:}

Baseline characteristics of included patients $(n=594)$. Data presented as number (\%); mean (+/- standard deviation); or median (interquartile range).

\begin{tabular}{|l|l|}
\hline Variable & Value \\
\hline Age, years & $67(+/-10)$ \\
\hline Male sex & $509(86 \%)$ \\
\hline
\end{tabular}


Sami, et al. 10

\begin{tabular}{|c|c|}
\hline Body mass index & $30.0(+/-4.9)$ \\
\hline $\begin{array}{l}\text { Length of Barrett's, cm (Prague M) } \\
\text { - Patients with long segment BE }(\geq 3 \\
\quad \mathrm{cm})\end{array}$ & $\begin{array}{l}4(2-6) \\
385(65.0 \%)\end{array}$ \\
\hline $\begin{array}{l}\text { Hiatal hernia presence } \\
\text { - Length of hiatal hernia, } \mathrm{cm}\end{array}$ & $\begin{array}{l}492(82.8 \%) \\
3(2-5)\end{array}$ \\
\hline $\begin{array}{l}\text { Pre-RFA baseline histology: } \\
\text { - } \quad \text { Non-dysplastic BE } \\
\text { - Indefinite for dysplasia } \\
\text { - } \quad \text { Low grade dysplasia } \\
\text { - } \quad \text { High grade dysplasia } \\
\text { - } \quad \text { Cancer }\end{array}$ & $\begin{array}{l}62(10.4 \%) \\
21(3.5 \%) \\
121(20.4 \%) \\
292(49.2 \%) \\
98(16.5 \%)\end{array}$ \\
\hline $\begin{array}{l}\text { Pre-RFA endoscopic resection } \\
\text { - Lesion histology: } \\
\text { - Non-dysplastic BE } \\
\text { - Indefinite for dysplasia } \\
\text { - Low grade dysplasia } \\
\text { - High grade dysplasia } \\
\text { - Cancer } \\
\text { i. Stage T1a; T1b; uncertain }\end{array}$ & $\begin{array}{l}326(54.9 \%) \\
48(14.7 \%) \\
1(0.3 \%) \\
53(16.3 \%) \\
142(43.6 \%) \\
82(25.1 \%) \\
78(95.1 \%) ; 3(3.7 \%), 1(1.2 \%)\end{array}$ \\
\hline $\begin{array}{l}\text { Index RFA device used } \\
\text { - Circumferential }\end{array}$ & 302 (51.3\%) \\
\hline
\end{tabular}




\begin{tabular}{|l|l|}
\hline- Focal & $287(48.7 \%)$ \\
\hline Number RFA sessions needed to CRIM & $2(1-3)$ \\
$-1-4$ & $554(93.3 \%)$ \\
$-5-8$ & $40(6.7 \%)$ \\
\hline
\end{tabular}

BE, Barrett's esophagus; RFA, radiofrequency ablation; CRIM, complete remission of intestinal metaplasia

151 subjects developed recurrent BE (Table 2) over a median (IQR) follow up of 2.8 (1.44.4) years.

Table 2: Baseline characteristics of recurrent cases $(n=151)$. Data presented as number (\%).

\begin{tabular}{|l|l|}
\hline Recurrence parameter & Value \\
\hline Location & $112(74.2 \%)$ \\
$-\quad$ Gubultroesophageal junction: & $39(25.8 \%)$ \\
\hline Histology: overall, tubular esophagus, GEJ & \\
$-\quad$ Non-dysplastic BE & $104(68.9 \%), 21(13.9 \%), 83(55 \%)$ \\
$-\quad$ Indefinite for dysplasia & $3(2.0 \%), 1(0.7 \%), 2(1.3 \%)$ \\
$-\quad$ Low grade dysplasia & $18(11.9 \%), 8(5.3 \%), 10(6.6 \%)$ \\
$-\quad$ High grade dysplasia & $12(7.9 \%), 4(2.6 \%), 8(5.3 \%)$ \\
$-\quad$ Cancer & $14(9.3 \%), 5(3.3 \%), 9(6.0 \%)$ \\
& $8(5.3 \%), 2(1.3 \%), 6(4.0 \%)$ \\
\hline
\end{tabular}


Sami, et al. 12

\begin{tabular}{|c|c|}
\hline $\begin{array}{l}\text { - } \quad \text { Stage T1a } \\
\text { - } \quad \text { Stage T1b } \\
\text { - } \quad \text { Stage T2 }\end{array}$ & $\begin{array}{l}4(2.6 \%), 2(1.3 \%), 2(1.3 \%) \\
2(1.3 \%), 1(0.65 \%), 1(0.65 \%)\end{array}$ \\
\hline Recurrence treated $\dagger$ & $108(71.5 \%)$ \\
\hline $\begin{array}{l}\text { Recurrence treatment modality: } \\
\text { - Endoscopic resection } \\
\text { - } \text { Ablation } \\
\text { - Endoscopic resection + ablation } \\
\text { - Endoscopic resection + chemo- } \\
\text { radiation } \\
\text { - Radiation only }\end{array}$ & $\begin{array}{l}10(9.3 \%) \\
83(76.9 \%) \\
13(12.0 \%) \\
1(0.9 \%) \\
1(0.9 \%)\end{array}$ \\
\hline $\begin{array}{l}\text { Recurrence treatment outcome } \\
\begin{array}{l}\text { - } \\
\text { - }\end{array} \text { OnIM achieved } \\
\text { - } \text { Lost to follow up } \\
\text { - Esophagectomy +/- chemo-radiation } \\
\text { - Radiation therapy only }\end{array}$ & $\begin{array}{l}73(67.6 \%) \\
25(23.1 \%) \\
4(3.7 \%) \\
4(3.7 \%) \\
2(1.9 \%)\end{array}$ \\
\hline
\end{tabular}

†Recurrence not treated in $43(28.5 \%)$ patients $(n=27$ awaiting treatment at the time of analysis; $n=7$ lost to follow up; $n=1$ deceased/lung cancer; $n=3$ no intestinal metaplasia on follow up; $n=5$ surveillance only). GEJ, gastroesophageal junction; BE, Barrett's esophagus; CRIM, complete remission of intestinal metaplasia.

Recurrence incidence and timeline 
The annual incidence rates of any recurrence, dysplastic recurrence, and HGD/cancer recurrence for the entire cohort are shown in Table 3.

Table 3: Annual incidence rates of recurrent BE after complete remission of intestinal metaplasia..

\begin{tabular}{|c|l|l|l|}
\hline Patient group & any & Dysplastic & HGD/cancer \\
recurrence & recurrence & recurrence \\
\hline All recurrences in entire study cohort $(n=594)$ & $9.6 \%$ & $2.8 \%$ & $1.6 \%$ \\
\hline Excluding NDBE recurrence at GEJ ( $n=594)$ & $4.3 \%$ & $2.8 \%$ & $1.6 \%$ \\
\hline Stratified by baseline histology pre-RFA & & & \\
\hline$-\quad$ NDBE/ID subgroup ( $n=83)$ & $5.2 \%$ & $0.7 \%$ & $0.7 \%$ \\
\hline$-\quad$ LGD subgroup (n=121) & $5.9 \%$ & $0.9 \%$ & $0.9 \%$ \\
\hline$-\quad$ HGD/cancer subgroup $(n=390)$ & $12.4 \%$ & $4.3 \%$ & $2.3 \%$ \\
\hline
\end{tabular}

HGD, high grade dysplasia; NDBE, non-dysplastic Barrett's esophagus; GEJ, gastroesophageal junction; RFA, radiofrequency ablation; ID, indefinite for dysplasia; LGD, low grade dysplasia; HGD, high grade dysplasia.

The recurrence hazard rate remained constant over the follow-up duration $(p=0.74)$ with $19 \%$ risk within 2 years and an additional $49 \%$ risk over the next 8.6 years. The recurrence hazard rate of dysplasia and HGD/Cancer while lower, also remained constant ( $p=0.94$ and $p=0.88$, respectively) over the duration of follow up (Figure 2). When NDBE recurrences at the GEJ were excluded, the recurrence hazard rate of any BE, dysplastic 
$\mathrm{BE}$, and HGD/cancer continued to remain constant over the follow up duration $(p=0.94$, 0.88, and 0.94, respectively) (Supplementary Figure 1).

Similarly, when stratified by baseline histology pre-RFA, the hazard rate of any recurrence remained constant over the follow-up duration in all the 3 groups of NDBE/ID $(p=0.15)$, LGD $(p=0.20)$, and HGD/cancer $(p=0.91)$ (Figure 3$)$. The hazard rate of dysplastic recurrence also remained constant in all the 3 groups of NDBE/ID $(p=0.62)$, LGD $(p=0.74)$, and HGD/cancer $(p=0.87)$ (Figure 4). Recurrence rates were higher in those treated for $\mathrm{HGD} /$ cancer.

\section{Recurrence location}

BE recurred at the GEJ in $74.2 \%(n=112)$ of subjects and in the tubular esophagus in $25.8 \%(n=39)($ Table 2). $75.9 \%(n=85)$ of recurrences at the GEJ were non-dysplastic and all of those were non-visible and only detected on random biopsies from the GEJ. 24.1\% $(n=27)$ of recurrences at the GEJ were dysplastic. Of these, $59.3 \%(n=16)$ were visible endoscopically (Cancer $n=9$, HGD $n=5$, LGD $n=2)$ and $40.7 \%(n=11)$ were non-visible ( $H G D n=3, L G D n=8$ ) and only detected on random biopsies of the GEJ.

$82.1 \%(n=32)$ of recurrences in the tubular esophagus were visible endoscopically and $84.4 \%$ of those were detected within $5 \mathrm{~cm}$ of the GEJ (Figure $5 A) .17 .9 \%(n=7)$ were nonvisible and only detected on random biopsies of the neo-squamous epithelium (Figure 5B) (five were subsquamous and 2 had no mention of columnar mucosa on the endoscopy report, but histology showed IM with no squamous epithelium in the specimen

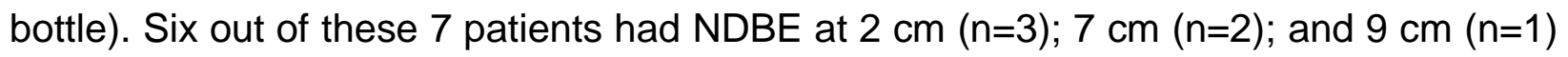


from the GEJ. One patient had LGD at $4 \mathrm{~cm}$ from the GEJ. Therefore, the overall yield of random biopsy sampling for NDBE recurrence was only $1.0 \%(6 / 594)$ for any recurrence, and $0.2 \%(1 / 594)$ for dysplastic recurrence.

\section{Predictors of recurrence}

Baseline HGD/cancer but not LGD, predicted any recurrence (HR 1.95, 95\%Cl 1.07-3.56; $\mathrm{p}=0.029$ ) (Supplementary Table 1 ) as well as dysplastic recurrence ( $\mathrm{HR} 4.81,95 \% \mathrm{Cl}$ 1.21-19.18; $p=0.026)$ (Supplementary Table 2).

\section{Supplementary Table 1:}

Multivariable model for any recurrence after complete remission of intestinal metaplasia with radiofrequency ablation.

\begin{tabular}{|l|l|l|l|}
\hline Variable & $\begin{array}{l}\text { Hazard } \\
\text { Ratio }\end{array}$ & $\begin{array}{l}\text { R5\% Hazard } \\
\text { Rimits }\end{array}$ & P value \\
\hline Age at CRIM & 1.01 & $0.99-1.03$ & 0.211 \\
\hline Male sex & 0.83 & $0.53-1.29$ & 0.398 \\
\hline Length of Barrett's cm (Prague M) & 1.03 & $0.97-1.10$ & 0.368 \\
\hline Presence of hiatal hernia & 1.62 & $0.93-2.82$ & 0.087 \\
\hline Baseline high grade dysplasia/cancer & 1.95 & $1.07-3.56$ & 0.029 \\
\hline Baseline low grade dysplasia & 1.01 & $0.52-1.97$ & 0.971 \\
\hline Endoscopic resection Pre-RFA & 1.17 & $0.78-1.77$ & 0.448 \\
\hline Use of circumferential RFA device & 1.17 & $0.75-1.83$ & 0.495 \\
\hline
\end{tabular}


Sami, et al. 16

\begin{tabular}{|l|l|l|l|}
\hline Variable & $\begin{array}{l}\text { Hazard } \\
\text { Ratio }\end{array}$ & $\begin{array}{l}\text { 95\% Hazard } \\
\text { Ratio Confidence } \\
\text { Limits }\end{array}$ & P value \\
\hline $\begin{array}{l}\text { Number of RFA sessions needed to } \\
\text { CRIM }\end{array}$ & 1.07 & $0.93-1.23$ & 0.344 \\
\hline $\begin{array}{l}\text { Use of other adjuvant ablation } \\
\text { techniques }\end{array}$ & 1.22 & $0.87-1.72$ & 0.242 \\
\hline
\end{tabular}

CRIM, complete remission of intestinal metaplasia; RFA, radiofrequency ablation.

\section{Supplementary Table 2:}

Multivariable model for dysplastic recurrence after complete remission of intestinal metaplasia with radiofrequency ablation.

\begin{tabular}{|c|c|c|c|}
\hline Parameter & $\begin{array}{l}\text { Hazard } \\
\text { Ratio }\end{array}$ & $\begin{array}{l}\text { 95\% Hazard } \\
\text { Ratio Confidence } \\
\text { Limits }\end{array}$ & P value \\
\hline Age at CRIM & 1.02 & $0.98-1.05$ & 0.381 \\
\hline Male sex & 1.77 & $0.58-5.39$ & 0.317 \\
\hline Length of Barrett's cm (Prague M) & 1.08 & $0.97-1.21$ & 0.165 \\
\hline Presence of hiatal hernia & 2.02 & $0.54-7.62$ & 0.298 \\
\hline Baseline high grade dysplasia/cancer & 4.81 & $1.21-19.18$ & 0.026 \\
\hline Baseline low grade dysplasia & 0.90 & $0.17-4.83$ & 0.906 \\
\hline Endoscopic resection Pre-RFA & 0.76 & $0.38-1.51$ & 0.433 \\
\hline
\end{tabular}




\begin{tabular}{|l|l|l|l|}
\hline Use of circumferential RFA device & 1.58 & $0.65-3.82$ & 0.313 \\
\hline $\begin{array}{l}\text { Number of RFA sessions needed to } \\
\text { CRIM }\end{array}$ & 1.09 & $0.85-1.38$ & 0.508 \\
\hline $\begin{array}{l}\text { Use of other adjuvant ablation } \\
\text { techniques }\end{array}$ & 1.92 & $1.04-3.57$ & 0.039 \\
\hline
\end{tabular}

CRIM, complete remission of intestinal metaplasia; RFA, radiofrequency ablation.

\section{Discussion}

\section{Principal findings}

In this large multicenter international cohort study, recurrence rates of NDBE, any dysplasia, and HGD/cancer following initial CRIM after RFA did not appear to plateau over the first 5-6 years of follow up, suggesting that continued yearly surveillance remains important, arguing against extending surveillance intervals at present, particularly in those with HGD/cancer at baseline. The majority of recurrences (74.2\%) developed at the GEJ and approximately a quarter of those were dysplastic, of which a significant proportion $(40.7 \%)$ were non-visible endoscopically. Most (84.4\%) visible recurrences in the tubular esophagus were located within $5 \mathrm{~cm}$ of the GEJ. Finally, the yield of tubular esophageal biopsies in the absence of visible recurrence was very low for NDBE (1.0\%) and 
dysplastic $B E(0.2 \%)$ which suggests that the requirement for random biopsies of the neosquamous epithelium in the absence of visible recurrence may need to be re-evaluated.

A recent modelling study based on data from US and UK RFA registries suggested surveillance endoscopies at one and three years after CRIM for patients with baseline LGD and endoscopies at three months, six months, one year, and then annually thereafter for those with baseline HGD or cancer. ${ }^{11}$ The surveillance intervals were estimated only to a limit of five years to avoid extrapolation beyond available data. Therefore, there is a lack of clarity from current literature with regards to both the need for and the yield of surveillance beyond this time. Moreover, the latter model's estimates were based on dysplastic recurrences only and did not account for the non-dysplastic (NDBE) ones. NDBE recurrences following CRIM may require therapy as those could represent an incompletely treated or missed prevalent disease and may still have neoplastic potential if left untreated. ${ }^{5}$ When NDBE recurrences at the GEJ were excluded (given concern that this may represent IM of the cardia rather than true BE recurrence ${ }^{13}$ ), the recurrence hazard rate across all grades of dysplasia remained constant over the duration of follow up (table 3 and supplementary figure 1). Our data suggests that continued surveillance beyond 5 years remains necessary. The recurrence rate in patients with LGD at baseline also remained constant over time, suggesting that long term surveillance is warranted in that group as well.

The number of endoscopies required to define CRIM remains a subject of debate. Intestinal metaplasia is known to be patchy and may be missed on random biopsies. ${ }^{14}$ Therefore, two endoscopies with biopsies may be required to confidently rule out the 
presence of IM. ${ }^{14}$ To our knowledge, there are no data comparing 2 vs. more negative endoscopies on the recurrence rates on follow up and this may be an area for further research. None of the three studies that defined CRIM on 2 negative endoscopies ${ }^{6-8}$ evaluated the variation in the incidence of recurrence over time across all grades of dysplasia. In one single center study, $20 \%$ of patients had NDBE at baseline and no recurrences were reported after 3 years implying little benefit from surveillance beyond 3 years post CRIM. ${ }^{6}$ This is in contrast to data from our study demonstrating that recurrence rate remained constant after 3 years.

Previously, two smaller, single center studies reported that the majority of dysplastic recurrences developed in the gastric cardia and the majority of those were non-visible. ${ }^{10}$ 15 Eighty percent of recurrences in the tubular esophagus were visible endoscopically 10 and random biopsies $>1 \mathrm{~cm}$ proximal to the GEJ had no yield for any recurrence. ${ }^{15}$ Data from the current study from a larger multicenter cohort showed that $82 \%$ of tubular esophageal recurrences were visible and the yield of random biopsies $>1 \mathrm{~cm}$ proximal to the GEJ was extremely low $(1.2 \%$ yield for any recurrence and $0.2 \%$ for dysplastic recurrence). The true rate of non-visible, sub-squamous recurrence remains hard to measure, but is likely to be rare based on current data. ${ }^{16}$ Volumetric laser endomicroscopy has been used to image post-RFA subsquamous glandular structures, but correlation with buried BE glands was poor in one study. ${ }^{17}$ More cost-effective and standardized imaging and sampling techniques are required in order to evaluate this outcome in a more systematic and precise manner. Moreover, data on the natural history of these recurrences are required.

\section{Study strengths and limitations}


This study has several strengths. We evaluated a large sample of patients over a long follow up duration, which are important factors to achieve more precise estimates of the recurrence rates. Data were collected from multiple expert large volume centers located both in the UK and USA, which strengthens the validity and generalizability of our results. Furthermore, the vast majority of our patients $(90 \%)$ received RFA for dysplastic BE which is representative of current clinical practice guidelines in contrast to several other studies of US populations where a large proportion of patients (up to $46 \%)^{11}$ have NDBE and therefore do not require RFA based on current evidence. ${ }^{2}$ Databases were prospectively maintained in all participating centers in order to minimize selection and recall biases. While centralized pathology was not utilized, all centers had dedicated expert gastrointestinal pathologists reading all BE histology.

The study also has limitations. Biopsy sampling techniques and forceps size used were not standardized across centers and may therefore result in sampling error and case ascertainment bias. We attempted to minimize the latter by implementing a more conservative definition of CRIM with 2 negative endoscopies and biopsies for IM from both the GEJ and tubular esophagus. The multicenter nature of the study with different operators at centers of expertise, makes the study susceptible to variation in practices, but also helps to make our data more representative of real world practice. One caveat to the latter is that our study sample comes from tertiary referral centers, which may not be representative of results in community practice. However, the overwhelming number of these procedures are currently performed in settings similar to ours based on current society guidelines. ${ }^{2}$

\section{Conclusions and implications for clinical practice}


This study demonstrates that the recurrence hazard rate of recurrent NDBE, dysplastic $\mathrm{BE}$, and $\mathrm{HGD} /$ cancer remained constant over time during surveillance in patients who achieved CRIM after RFA when strict criteria for the definition of CRIM are applied. This suggests that diligent long term (at least) yearly endoscopic surveillance remains important in these patients. The majority of all recurrences developed at the GEJ and a significant proportion of dysplastic recurrences were non-visible endoscopically. This reenforces the need for careful imaging and sampling of the GEJ despite the absence of any visible lesions. On the other hand, the majority of recurrences in the tubular esophagus are visible endoscopically and the yield of random biopsy sampling in the absence of visible lesions was very low in expert centers. These findings may need to be replicated in non-expert centers before further conclusions can be made with regards to the cost-effectiveness of this practice. 


\section{Acknowledgements:}

Ms. Lyndsay Busby for assistance in formatting manuscript.

\section{Disclosures:}

Prasad G lyer: Research funding from Exact Sciences, C2 Therapeutics, Medtronic, Nine Point Medical

Consulting: C2 Therapeutics, Symple Surgical

Grant support: None

\section{Author contributions:}

Sarmed S. Sami: contributed to the design and coordination of the study; acquisition of data; analysis; interpretation of data; and drafted the manuscript.

Adharsh Ravindran: contributed to the acquisition of data; and critical revision of the manuscript for important intellectual content.

Allon Kahn: contributed to the acquisition of data; and critical revision of the manuscript for important intellectual content.

Diana Snyder: contributed to the acquisition of data; and critical revision of the manuscript for important intellectual content.

Jose Santiago: contributed to the acquisition of data; and critical revision of the manuscript for important intellectual content.

Jacobo Ortiz-Fernandez-Sordo: contributed to the acquisition of data; and critical revision of the manuscript for important intellectual content. 
Keith Tan: $\quad$ contributed to the acquisition of data; and critical revision of the manuscript for important intellectual content.

Michael Heckman: contributed to the acquisition of data; and critical revision of the manuscript for important intellectual content.

Ross Dierkhising: contributed to the analysis; interpretation of data; and critical revision of the manuscript for important intellectual content.

Michele Johnson: contributed to the acquisition of data; and critical revision of the manuscript for important intellectual content.

Ramona Lansing: contributed to the acquisition of data; and critical revision of the manuscript for important intellectual content.

Kenneth Wang: contributed to the design of the study; interpretation of data; and critical revision of the manuscript for important intellectual content.

Krish Ragunath: contributed to the study supervision; interpretation of data; and critical revision of the manuscript for important intellectual content.

Massimiliano di Pietro: contributed to the study supervision; interpretation of data; and critical revision of the manuscript for important intellectual content.

Herbert Wolfsen: contributed to the study supervision; interpretation of data; and critical revision of the manuscript for important intellectual content.

Francisco Ramirez: contributed to the study supervision; interpretation of data; and critical revision of the manuscript for important intellectual content.

David Fleischer: contributed to the study supervision; interpretation of data; and critical revision of the manuscript for important intellectual content. 
Cadman Leggett: contributed to the design of the study; interpretation of data; and critical revision of the manuscript for important intellectual content.

David Katzka: contributed to the design of the study; interpretation of data; and critical revision of the manuscript for important intellectual content.

Prasad G. lyer: $\quad$ contributed to the conception, design and supervision of the study; acquisition of data; analysis; interpretation of data; and critical revision of the manuscript for important intellectual content. 


\section{References}

1. Desai TK, Krishnan K, Samala N, et al. The incidence of oesophageal adenocarcinoma in non-dysplastic Barrett's oesophagus: a meta-analysis. Gut 2012;61:970-6.

2. Shaheen NJ, Falk GW, lyer PG, et al. ACG Clinical Guideline: Diagnosis and Management of Barrett's Esophagus. Am J Gastroenterol 2016;111:30-50.

3. Krishnamoorthi R, Singh S, Ragunathan K, et al. Risk of recurrence of Barrett's esophagus after successful endoscopic therapy. Gastrointest Endosc 2016;83:1090-1106 e3.

4. Fujii-Lau LL, Cinnor B, Shaheen N, et al. Recurrence of intestinal metaplasia and early neoplasia after endoscopic eradication therapy for Barrett's esophagus: a systematic review and meta-analysis. Endosc Int Open 2017;5:E430-e449.

5. Sawas T, lyer PG, Alsawas M, et al. Higher Rate of Barrett's Detection in the First Year After Successful Endoscopic Therapy: Meta-analysis. Am J Gastroenterol 2018;113:959971.

6. Komanduri S, Kahrilas PJ, Krishnan K, et al. Recurrence of Barrett's Esophagus is Rare Following Endoscopic Eradication Therapy Coupled With Effective Reflux Control. Am J Gastroenterol 2017;112:556-566.

7. Gupta M, lyer PG, Lutzke L, et al. Recurrence of esophageal intestinal metaplasia after endoscopic mucosal resection and radiofrequency ablation of Barrett's esophagus: results from a US Multicenter Consortium. Gastroenterology 2013;145:79-86.e1.

8. Vaccaro BJ, Gonzalez S, Poneros JM, et al. Detection of intestinal metaplasia after successful eradication of Barrett's Esophagus with radiofrequency ablation. Dig Dis Sci 2011;56:1996-2000.

9. Cotton CC, Wolf WA, Overholt BF, et al. Late Recurrence of Barrett's Esophagus After Complete Eradication of Intestinal Metaplasia is Rare: Final Report From Ablation in Intestinal Metaplasia Containing Dysplasia Trial. Gastroenterology 2017;153:681-688.e2. 
10. Guthikonda A, Cotton CC, Madanick RD, et al. Clinical Outcomes Following Recurrence of Intestinal Metaplasia After Successful Treatment of Barrett's Esophagus With Radiofrequency Ablation. Am J Gastroenterol 2017;112:87-94.

11. Cotton CC, Haidry R, Thrift AP, et al. Development of Evidence-Based Surveillance Intervals After Radiofrequency Ablation of Barrett's Esophagus. Gastroenterology 2018;155:316-326 e6.

12. von Elm E, Altman DG, Egger M, et al. Strengthening the Reporting of Observational Studies in Epidemiology (STROBE) statement: guidelines for reporting observational studies. Bmj 2007;335:806-8.

13. Spechler SJ, Zeroogian JM, Antonioli DA, et al. Prevalence of metaplasia at the gastrooesophageal junction. Lancet 1994;344:1533-6.

14. Jones TF, Sharma P, Daaboul B, et al. Yield of intestinal metaplasia in patients with suspected short-segment Barrett's esophagus (SSBE) on repeat endoscopy. Dig Dis Sci 2002;47:2108-11.

15. Cotton CC, Wolf WA, Pasricha S, et al. Recurrent intestinal metaplasia after radiofrequency ablation for Barrett's esophagus: endoscopic findings and anatomic location. Gastrointest Endosc 2015;81:1362-9.

16. Gray NA, Odze RD, Spechler SJ. Buried metaplasia after endoscopic ablation of Barrett's esophagus: a systematic review. Am J Gastroenterol 2011;106:1899-908.

17. Swager AF, Boerwinkel DF, de Bruin DM, et al. Detection of buried Barrett's glands after radiofrequency ablation with volumetric laser endomicroscopy. Gastrointest Endosc 2016;83:80-8.

\section{Figure Legends:}


Figure 1: Patient flowchart. CRIM, complete remission of intestinal metaplasia. ${ }^{*}$ Recurrence not treated in 43 patients ( $n=27$ awaiting treatment at the time of analysis; $n=7$ lost to follow up; $n=1$ deceased/lung cancer; $n=3$ no intestinal metaplasia on follow up; $n=5$ surveillance only).

Figure 2: The timeline of recurrent Barrett's esophagus (any recurrence; dysplastic recurrence; and high grade dysplasia (HGD)/cancer recurrence) following complete remission of intestinal metaplasia (CRIM).

Figure 3: The timeline of any recurrence stratified by baseline histology prior to radiofrequency ablation. NDBE: non-dysplastic Barrett's esophagus; LGD: low grade dysplasia; HGD: high grade dysplasia.

Figure 4: The timeline of dysplastic recurrences stratified by baseline histology prior to radiofrequency ablation. NDBE: non-dysplastic Barrett's esophagus; LGD: low grade dysplasia; HGD: high grade dysplasia.

Figure 5: The location of visible (panel A) and non-visible (panel B) recurrences in the tubular esophagus (blue cylinder). Histology of non-visible recurrences is also shown in panel B (NDBE: non-dysplastic Barrett's esophagus; LGD: low grade dysplasia).

Supplementary Figure 1: The timeline of recurrent Barrett's esophagus (any recurrence; dysplastic recurrence; and high grade dysplasia (HGD)/cancer recurrence) following complete remission of intestinal metaplasia (CRIM) and after excluding non-dysplastic recurrences at the gastroesophageal junction. 